\title{
Usability of the GPS Precise Point Positioning Technique in Marine Applications
}

\author{
R.M. Alkan ${ }^{1}$ and T. Öcalan ${ }^{2}$ \\ ${ }^{1}$ (Hitit University, Corum, Turkey \& Istanbul Technical University, Istanbul, Turkey) \\ ${ }^{2}$ (Yildiz Technical University, Istanbul, Turkey) \\ (E-mail: alkan@hitit.edu.tr)
}

\begin{abstract}
This study investigates the accuracy of an online Precise Point Positioning (PPP) service operated by the Geodetic Survey Division of Natural Resources Canada (NRCan), Canadian Spatial Reference System (CSRS)-PPP, by using single/dual-frequency Global Positioning System (GPS) data collected by dual-frequency geodetic-grade and Original Equipment Manufacturer (OEM) board type single-frequency GPS receivers. In this work, a kinematic test was carried out in Halic Bay (Golden Horn), Istanbul, Turkey, to assess the performance of the PPP method in a dynamic environment. Based on this study, it can be concluded that the coordinates estimated from the online CSRS-PPP service have a potential of about metre-level accuracy by processing single frequency data collected by an OEM receiver and about a decimetre to a few centimetres level accuracy by processing dual frequency data collected by a geodetic-grade receiver. In general, results show that the PPP technique has become a significant alternative to the conventional relative (differential) positioning techniques (i.e., Differential GPS (DGPS), Real-time Kinematic (RTK)). The technique does not suffer from the drawbacks of the DGPS technique and has potential to provide the same position accuracy without the requirement for a reference station. Consequently, it has been concluded that the PPP technique may be effectively used in marine applications due to its ease of use and provision of high accuracy, as well as being able to offer reduced field operational costs.
\end{abstract}
KEY WORDS
1. OEM GPS receiver.
2. PPP.
3. Web-based service.
4. Marine applications.

Submitted: 25 August 2012. Accepted: 4 April 2013. First published online: 9 May 2013.

1. INTRODUCTION. In recent years, to improve Global Positioning System (GPS) point positioning accuracy, new algorithms have been proposed. Precise Point Positioning (PPP) is one of the Global Navigation Satellite System (GNSS) analysis techniques in which no other observations from a reference station or a reference station network as used by conventional differential algorithms are required. The technique uses precise satellite orbit and clock information (produced by different 
organizations such as IGS, CODE, JPL), un-differenced code pseudoranges and/or carrier phase observations (HeBelbarth and Wanninger, 2010; Zumberge et al., 1997). In this technique, while metre level accuracies can be obtained by processing the data collected by the single frequency receiver, sub-metre, decimetre and even centimetre level accuracies can be obtained by using dual frequency receivers (Choy et al., 2007; Gao and Shen, 2002; Kouba and Héroux, 2001; Kouba, 2003; van Bree and Tiberius, 2012; Zumberge et al., 1997). To reach the highest accuracies in static and kinematic applications, long-term observations, including continuous dual-frequency carrierphase data are required. Marine kinematic positioning applications require decimetre to centimetre level accuracies, which can be provided by the PPP technique in postprocessing mode (Geng et al., 2010).

Users have various software alternatives for providing PPP-derived coordinates in static or kinematic mode. These can be classified as scientific software (i.e., Bernese, GIPSY/OASIS, WaPPP), in-house and web-based online PPP services (e.g., CSRSPPP, GAPS, APPS, magicGNSS). Conventionally, in order to process and analyse GPS data, GPS professionals have used many scientific and commercial software packages. Besides the high costs of these packages, users also require training and experience. Among these alternatives, web-based online PPP post-processing services have been preferred as they are user-friendly, free to access, without any requirement for software knowledge and provide quick solutions via e-mail and/or ftp. These solutions, as well as a variety of graphical analyses, are sent back to the users through e-mail (URL-1, 2012; URL-2, 2012). One of the most widely used web-based online PPP services worldwide is the Canadian Spatial Reference System (CSRS)-PPP, which was established by the Geodetic Survey Division of Natural Resource Canada (NRCan) in 2003. The flowchart of the CSRS-PPP service starts with submitting the observation file in RINEX format through its web site. Here, the user selects the mode of processing (static/kinematic) and reference system (ITRF/NAD83), and then the software estimates the point coordinates (BLH/XYZ/UTM) with their standard deviations. The service generates solutions in case L1 and L2 code pseudorange and carrier phase observations are available when dual frequency receivers are used. If the service uses single frequency data, only L1 code pseudorange solutions can be performed. CSRS-PPP service uses the precise satellite orbit, clock information, and antenna corrections from IGS. The users receive the results via e-mail. The observation and submitting date is significant for the accuracy of the resulting coordinates, because the service uses precise satellite orbits and clock products with different latencies, i.e., ultra rapid, rapid or final. This service has actively processed GLONASS data since 4 October 2011 and accepted Ocean Tidal Loading (OTL) correction files provided by users from 14 February 2012 (HeBelbarth and Wanninger, 2010; URL-2, 2012).

In recent years, due to the high-cost of dual frequency geodetic receivers, low-cost single-frequency Original Equipment Manufacturer (OEM) GPS receivers have been widely used in survey and navigation applications and scientific research. The cost of an OEM GPS receiver is less than 1,000 USD, sometimes just a few hundreds of USD. Several researchers have investigated the usability of such a system in surveying applications in static and kinematic modes and reported promising results (Abidin and Muchlas, 2005; Alkan et al., 2006; Alkan et al., 2008; Alkan and Saka, 2009; Cosser et al., 2004; Hill et al., 2001; Masella et al., 1997; Masella, 1999; Rizos et al., 1998; Saeki and Hori, 2006; Schwieger and Gläser, 2005; Söderholm, 2005). 
The aim of this study is to investigate the PPP-derived coordinate accuracies estimated by web-based online PPP services using the geodetic grade dual and OEM type low-cost single frequency receivers in the marine environment. For this, a field test has been conducted in Halic Bay (Golden Horn), Istanbul, Turkey. In this paper, the test procedures and their results are discussed.

2. MATHEMATICAL MODELLING OF PPP. PPP techniques have developed into most effective methods of GPS positioning. One of the main drivers for this has been to achieve accurate positioning in large scale application areas without requiring dedicated reference stations. However, traditional PPP also requires longer convergence time to achieve centimetre-level accuracy. Thus, rapid and real time applications of PPP are currently limited. So, in recent years, many mathematical models have been investigated and developed to shorten the convergence time and improve the accuracy in PPP solutions for fixing integer ambiguities (Kouba and Héroux, 2001; Bisnath and Gao, 2007; Li and Zhang, 2012).

PPP uses undifferenced ionospheric-free both carrier-phase $(\Phi)$ and/or code pseudorange (P) observations collected by dual-frequency receivers for data processing. This technique provides precise positioning by using precise ephemeris and clock products provided by IGS and other organizations.

By applying a set of corrections to these products, PPP performance may be further improved. The other corrections may be classified as: satellite effects (satellite antenna offsets and phase wind-up), site displacement effect (solid earth tides, polar tides, ocean loading, earth rotation parameters) and compatibility considerations (product formats, reference frames, receiver antenna phase centre offsets, modelling/ observation conventions) (El-Rabbany, 2006; Weston and Schwieger, 2010; Zumberge et al., 1997; Kouba and Héroux, 2001; Kouba 2009).

As stated in both Zumbarge et al. (1997) and Kouba and Heroux (2001), the ionospheric-free combinations of dual-frequency GPS pseudorange $(P)$ and carrierphase observations $(\Phi)$ depend on the user position, clock, troposphere and ambiguity parameters to reduce the following simplified observation equations:

$$
\begin{gathered}
P=\rho+c(d T-d t)+T_{r}+\varepsilon_{p} \\
\Phi=\rho+c(d T-d t)+T r+N \lambda+\varepsilon \Phi
\end{gathered}
$$

where

$\begin{array}{ll}P & \text { is the ionosphere-free combination of } \mathrm{P} 1 \text { and } \mathrm{P} 2 \text { pseudoranges } \\ & (P 3)=\left(2 \cdot 546 P_{1}-1 \cdot 546 P_{2}\right) \\ & \text { is the ionosphere-free combination of } \mathrm{L} 1 \text { and } \mathrm{L} 2 \text { carrier-phases } \\ & (L 3)=\left(2 \cdot 546 \lambda_{1} \Phi_{1}-1 \cdot 546 \lambda_{2} \Phi_{2}\right) \\ & \text { is the geometrical range computed as a function of satellite and station } \\ & \text { coordinates } \\ c & \text { is the vacuum speed of light } \\ d T & \text { is the station receiver clock offset from the GPS time } \\ d t & \text { is the satellite clock offset from the GPS time } \\ T_{r} & \text { is the signal path delay due to the neutral-atmosphere (primarily the } \\ & \text { troposphere) }\end{array}$




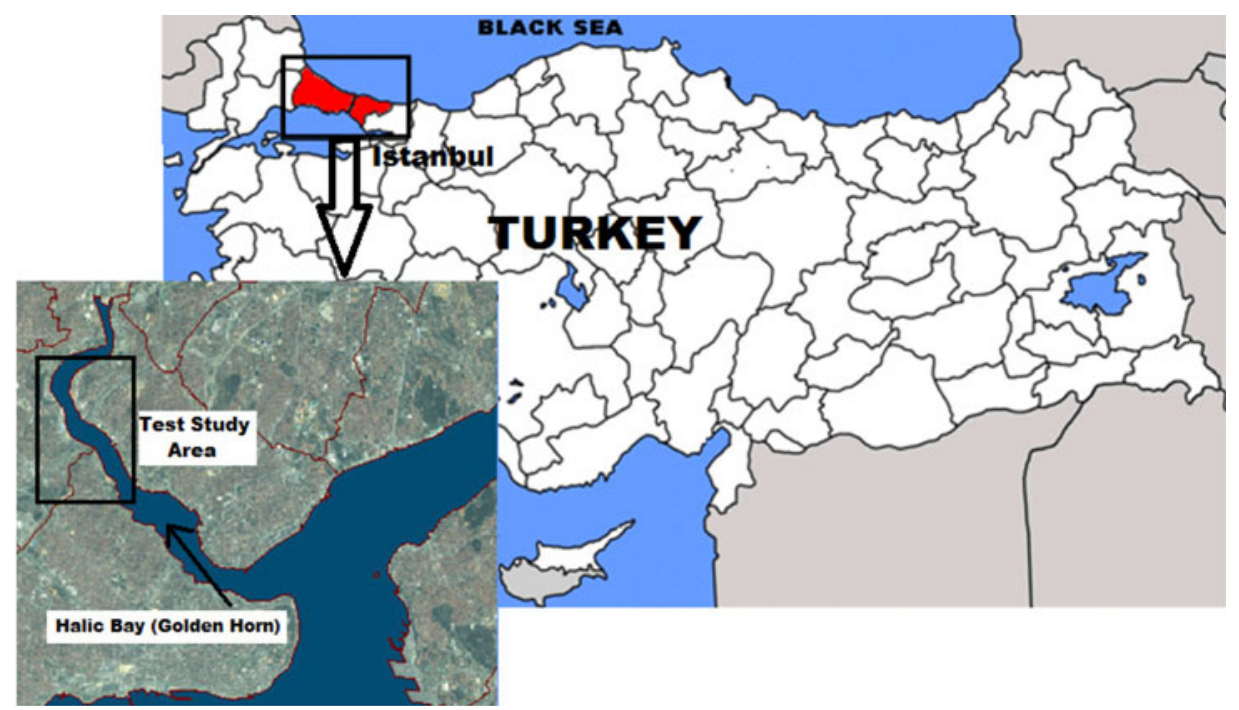

Figure 1. Location of the Test Study Area, Golden Horn, Istanbul.

$N$ is the non-integer ambiguity of the carrier-phase ionosphere-free combination

$\lambda_{1}, \lambda_{2}, \lambda$ are the of the carrier-phases L1, L2 and L3-combined $(10 \cdot 7 \mathrm{~cm})$ wavelengths, respectively

$\varepsilon_{P}, \varepsilon_{\Phi} \quad$ are the relevant measurement noise components, including multipath.

3. KINEMATIC TEST. In order to test the performance of the PPP technique in kinematic application, a field test was conducted in Halic Bay (Golden Horn), Istanbul in August 2009 (Figure 1).

In this study, two different types of GPS receivers, i.e., geodetic-grade dual frequency (Ashtech Z-Xtreme) and OEM type single-frequency (Ashtech AC12), were used (Figure 2). The high-end Ashtech Z-Xtreme receiver was chosen because all its performance characteristics are well known.

The Z-Xtreme receiver is designed to provide surveyors with cost-effective and centimetre-level accuracy positions in a variety of system configurations. The AC12 is a single frequency receiver capable of precise carrier phase output and the carrier phase measurements deliver high accuracy and flexibility for many applications including land and marine navigation, low-cost heading and attitude systems, deformation monitoring, asset and personnel tracking, relative navigation, automotive, military, and even golf course management. Some important features that are offered by the AC12 are:

- 12-channel continuous tracking with $1 \mathrm{~Hz}$ update rate

- Low power consumption (0.23 watts)

- User-defined and pre-defined datums

- Two-way serial port communications

- L1 frequency 


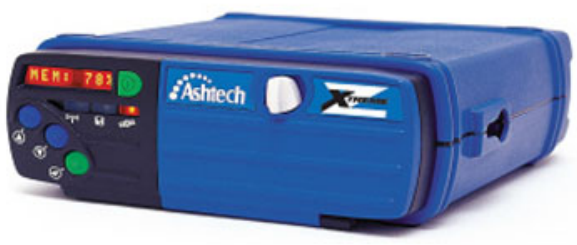

Ashtech Z-Xtreme

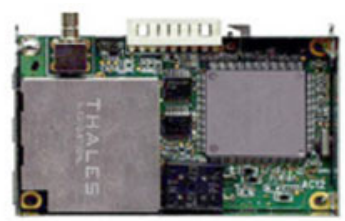

Ashtech AC12

Figure 2. Geodetic-grade receiver (left) and OEM receiver (right) used in this study.

- Raw data output (code and carrier)

- Precise carrier-phase tracking (carrier phase measurement accuracy is $3 \mathrm{~mm}$ )

- 1 PPS output accurate to better than 250 nanoseconds

More details about the receiver can be found in the manual (Thales, 2005).

To calculate the true coordinates of the vessel for each epoch using differential techniques, one geodetic-grade GPS receiver was stationed on a reference point with known coordinates on the shore. In the implementation phase of the kinematic test observation, a geodetic-grade GPS receiver (Ashtech Z-Xtreme) and OEM (Ashtech AC12) receiver were connected to a dual frequency GPS antenna via an antenna splitter. This provides collection of GPS data by both receivers under identical conditions. After the static initialization on shore, the GPS antenna was mounted to the vessel and the kinematic test was started (Figure 3). The measurements were performed along several survey profiles and data were collected for approximately 2 hours with a small vessel (Figure 4).

4. DATA PROCESSING STRATEGY AND PPP ANALYSIS. All collected GPS data were converted to the RINEX format and uploaded to the CSRS-PPP service on the next full day after the data collection day by using the service's interactive web page and choosing kinematic processing mode (Figure 5).

Some time after the submission, an e-mail was received from the service that contained a URL link and estimated PPP-derived coordinates with some additional information. The processing options handled in the estimation of the PPP-derived coordinates are given in Table 1. As can be seen from the table, the service has used code and carrier phase data for the geodetic-grade dual frequency receiver. Also, the service has produced an L1 code-only solution for the OEM type GPS receiver, because the submitted RINEX file contains only single-frequency data. The service used IGS rapid orbit data because the data were sent to the service next day after the collected data (i.e., 1 day latency).

The data collected by geodetic-grade receivers both on the vessel and stationed ashore were processed by the Leica Geosystems commercial GPS processing software, Leica Geo Office (LGO), to obtain reference trajectory. The PPP-derived coordinates from the online service for both geodetic-grade and OEM type receivers were compared with those of relative (differential) technique results epoch by epoch. The differences in position and ellipsoidal heights between the PPP-derived and relative solutions are shown in Figure 6. 


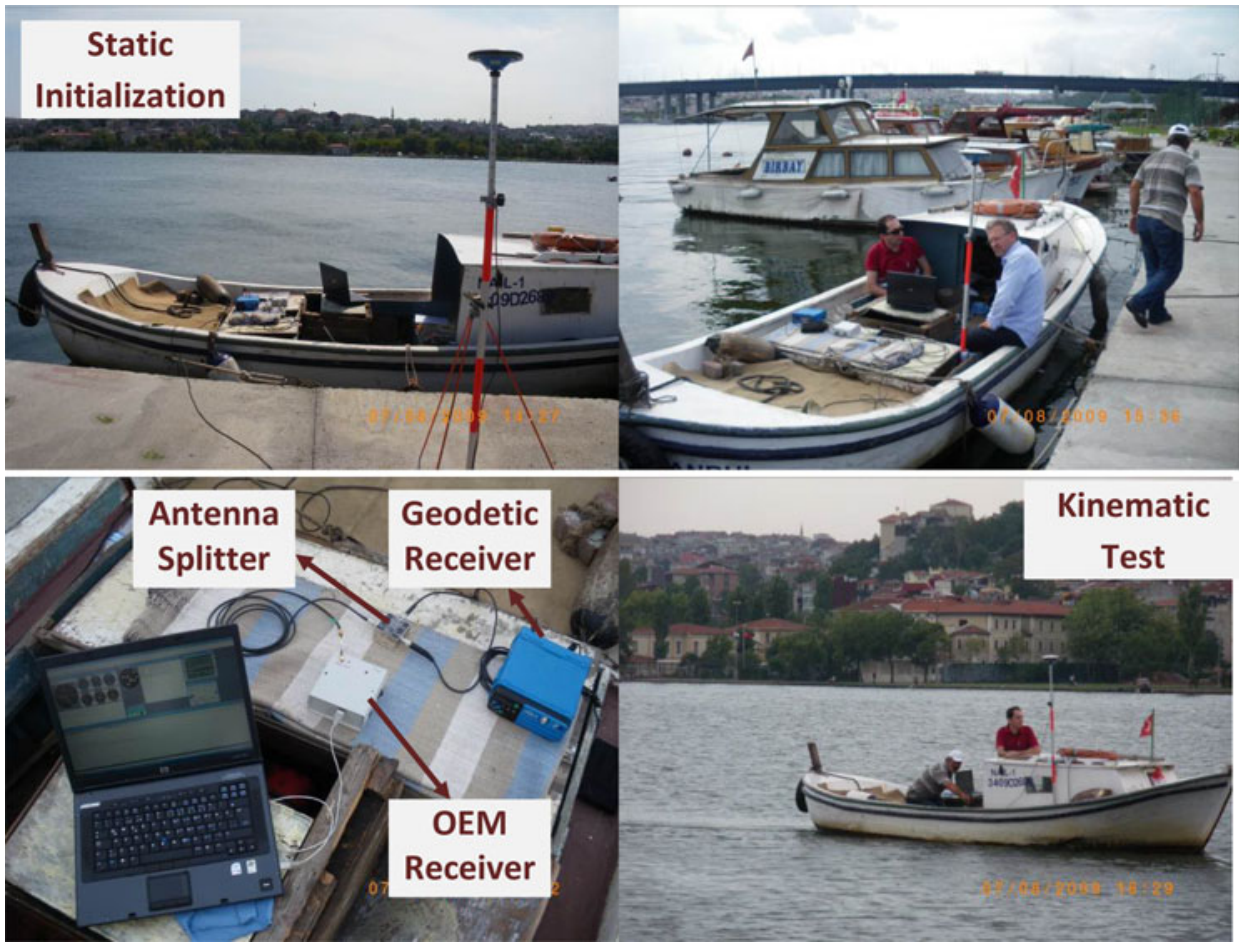

Figure 3. Kinematic Test in the Study Area.

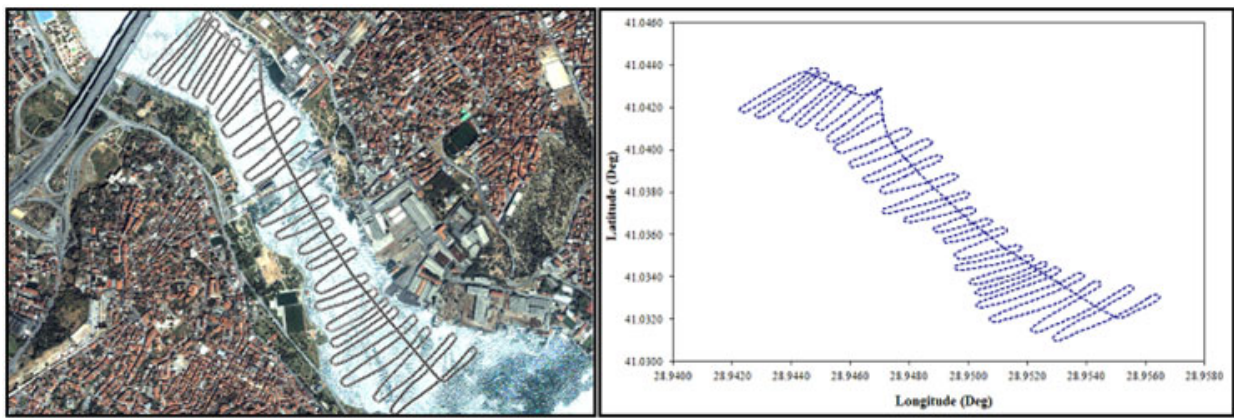

Figure 4. Vessel Trajectory.

It can be seen from Figure 6 that the positional accuracy reaches $15 \mathrm{~cm}$ and for height up to $25 \mathrm{~cm}$ for data from the geodetic-grade receiver processed by the PPP technique. At the same time, it can be seen that the results for PPP analyses obtained from the OEM GPS receiver were converged to the results evaluated by relative technique with metres-level accuracies. For the height component, less accurate results were obtained. The basic reason for the lower performance of the OEM receiver is due to the data collection with only the single L1 frequency signal. 


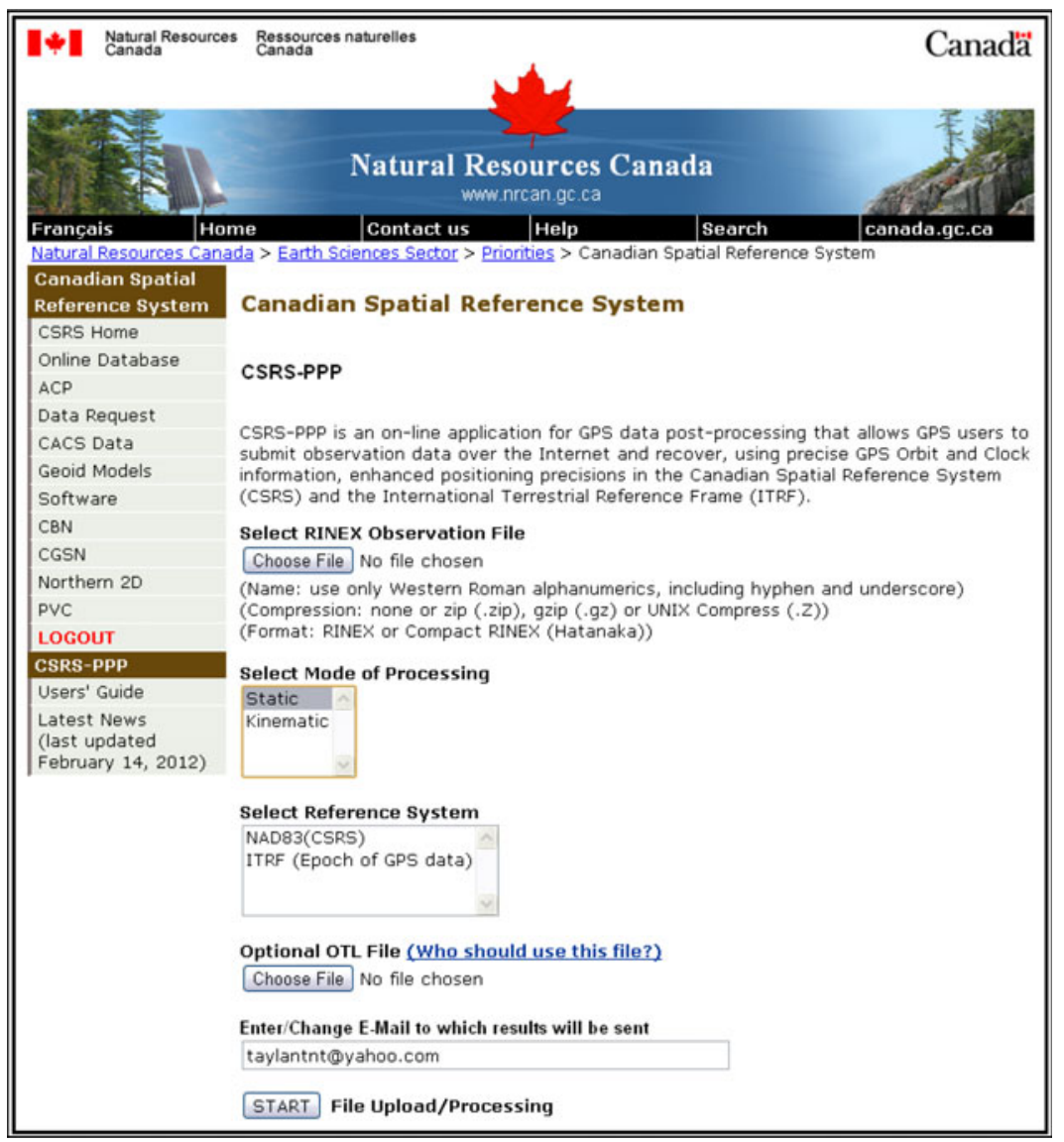

Figure 5. Online CSRS-PPP Service Web Site (as of June, 2012) (http://www.geod.nrcan.gc.ca/ online_data_e.php).

5. RESULTS AND DISCUSSION. In this study, the performance of the Precise Point Positioning (PPP) technique in marine applications was investigated using geodetic-grade and OEM GPS receivers through the online Canadian Spatial Reference System (CSRS)-PPP service. After the evaluation of the data obtained from the dual frequency receiver, centimetre to decimetre level position accuracies were achieved. Results obtained from the low-cost single frequency OEM GPS receiver were converged to the relative technique within metre-level accuracy. It was found that the obtained accuracy is good enough for many studies including hydrographical surveys, marine geodesy, navigation and oceanography.

Today, the PPP technique provides high point positioning accuracies by using a single receiver on the absolute positioning principle. Ease of application, low-cost opportunities and precision in point positioning are the main reasons for utilising the system in survey applications. PPP is thus a significant alternative to the classical relative (differential) positioning techniques.

The usability of the CSRS-PPP web-based online service has become popular with the advantages of user-friendly interface, being free-of charge, with no requirement for 
Table 1. PPP Processing Options for Kinematic Application.

\begin{tabular}{llll}
\hline & & \multicolumn{2}{c}{ Used Options } \\
\cline { 3 - 4 } Settings & Possible Options & $\begin{array}{l}\text { OEM } \\
\text { Receiver (L1) }\end{array}$ & $\begin{array}{l}\text { Geodetic Receiver } \\
\text { (L1\&L2) }\end{array}$ \\
\hline User Dynamics & Static/Kinematic & Kinematic & Kinematic \\
Observation Processed & Code/Code\&Phase & Code & Code\&Phase \\
Frequency Observed & L1/L1\&L2 & L1 & L3 \\
Satellite Orbits & Broadcast/Precise & Precise & Precise \\
Satellite Product Input & 15-Minute/5-Minute & 5-Minute & 5-Minute \\
Ionospheric Model & Broadcast/IONEX/ & IONEX & L1\&L2 \\
& L1\&L2 & & \\
Marker Coordinates & Fixed/Estimated & Estimated & Estimated \\
Troposphere Zenith Delay & Modelled/Estimated/ & Fixed & Estimated \\
& Fixed & & \\
Clock Interpolation & Yes/No & Yes & Yes \\
Parameter Smoothing & Yes/No & No & Yes \\
Reference Frame & ITRF/NAD83(CSRS) & ITRF & ITRF \\
Pseudorange Sigma (m) & A-Priori Estimate & $1 \cdot 000$ & $2 \cdot 000$ \\
Carrier Phase Sigma (m) & A-Priori Estimate & $0 \cdot 150$ & $0 \cdot 015$ \\
Cutoff Elevation (deg) & 0->90 Degrees & 10 & 10 \\
Pseudorange residuals $(\mathbf{m})$ & & $0 \cdot 88$ & $0 \cdot 43$ \\
Carrier phase residuals (cm) & & $0 \cdot 00$ & $0 \cdot 75$ \\
Satellite orbits & & Rapid & Rapid \\
Satellite clocks & & Rapid & Rapid \\
\hline
\end{tabular}
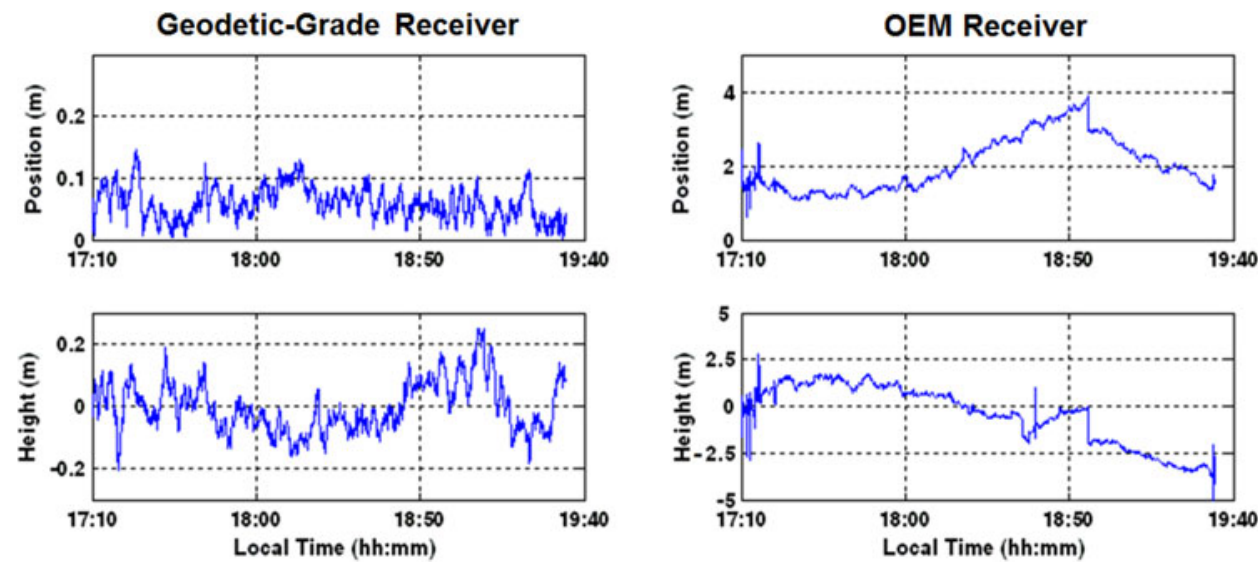

Figure 6. The differences between PPP-derived and relative solutions.

a license, knowledge of GPS processing software or the complex background to the technique. The user only needs a PC/mobile device with an internet connection and web browser. Moreover, the user may obtain coordinates only a few hours after submitting the collected GPS data to the service. The major drawback of the web service is that the system does not allow users to select the available options above the optimal options set by the system. 


\section{ACKNOWLEDGMENT}

The authors would like to thank to the Natural Resources Canada (NRCan), Geodetic Survey Division (Ressources naturelles Canada, Division des levés géodésiques) for giving permission to use their online CSRS-PPP service for this scientific study.

\section{REFERENCES}

Abidin, H.Z. and Muchlas, A. (2005). GPS Surveying Using Navigation Type Receivers. In: Proceedings of the South East Asia Survey Congress 2005, Bandar Seri Begawan, Brunei Darussalam, November $21-25,2005$.

Alkan, R.M., El-Rabbany, A. and Saka, M.H. (2006). Assessment of Low-Cost Garmin OEM GPS Receiver for Surveying Applications. Ontario Professional Surveyor, 49(4), 14-16.

Alkan, R.M., Saka, M.H., Kalkan, Y. and Şahin, M. (2008). Usability of Low-Cost L1 Frequency GPS Receivers in Surveying Applications. In: Proceedings of the Toulouse Space Show'08, European Navigation Conference (ENC-GNSS), Toulouse, France, April 22-25, 2008.

Alkan, R.M. and Saka, M.H. (2009). A Performance Analysis of Low-Cost GPS Receivers in Kinematic Applications. Journal of Navigation, 62 (4), 687-697.

Bisnath, S. and Gao, Y. (2007). Current State of Precise Point Positioning and Future Prospects and Limitations, Proceedings of Observing our Changing Earth. IAG Symopsium Series, 133, 615-624.

Choy, S.L., Zhang, K., Silcock, D. and Wu, F. (2007). Precise Point Positioning - A Case Study in Australia. In Proc. of Spatial Sciences Institute International Conference (SSC2007), Tasmania, Australia, May 2007, 192-202.

Cosser, E., Hill, C.J., Roberts, G.W., Meng, X., Moore, T. and Dodson, A.H. (2004). Bridge Monitoring with Garmin Handheld Receivers. In: Proc. of the 1st FIG International Symposium on Engineering Surveys for Construction Works and Structural Engineering, Nottingham, United Kingdom.

El-Rabbany, A. (2006). Introduction to GPS: The Global Positioning System. Second Edition, Artech House.

Gao, Y. and Shen, X. (2002). A New Method For Carrier-Phase-Based Precise Point Positioning. Navigation: Journal of Institute of Navigation, 49(2), 109-116.

Geng, J., Teferle, F.N., Meng, X. and Dodson, A.H. (2010). Kinematic Precise Point Positioning at Remote Marine Platforms. GPS Solutions, 14(4), 343-350.

HeBelbarth, A. and Wanninger, L. (2010). Performance of GNSS-PPP in Post-Processing Mode. Hydro 2010, Rostock-Warnemuende, Germany, November 2-5, 2010.

Hill, C.J., Moore, T. and Dumville, M. (2001). Carrier Phase Surveying with Garmin Handheld GPS Receivers. Survey Review, 36(280), 135-141.

Kouba, J. and Héroux, P. (2001). Precise Point Positioning Using IGS Orbit and Clock Products. GPS Solutions, 5(2), 12-28.

Kouba, J. (2003). A Guide to Using International GPS Service (IGS) Products. IGS Central Bureau, http:// igscb.jpl.nasa.gov/igscb/resource/pubs/GuidetoUsingIGSProducts.pdf), February 2003.

Kouba, J. (2009). A Guide to Using International GNSS Service (IGS) Products. IGS Central Bureau, http:// igscb.jpl.nasa.gov/components/usage.html), May 2009.

Li, X. and Zhang, X. (2012). Improving the Estimation of Uncalibrated Fractional Phase Offsets for PPP Ambiguity Resolution. The Journal of Navigation, 65(3), 513-529.

Masella, E. (1999). Achieving $20 \mathrm{~cm}$ Positioning Accuracy in Real Time Using GPS-the Global Positioning System. GEC Review, 14(1), 20-27.

Masella, E., Gonthier, M. and Dumaine, M. (1997). The RT-Star: Features and Performance of a Low-Cost RTK OEM Sensor. In: Proc. of the ION GPS'97, The International Technical Meeting of the Satellite Division of the ION, Kansas City, Missouri, 53-59.

Rizos, C., Han, S. and Han, X. (1998). Performance Analysis of a Single-Frequency, Low-Cost GPS Surveying System. In: Proc. of the 11th Int. Tech. Meeting of the Satellite Division of the US ION, GPS ION'98, Nashville, Tennessee, USA, 427-435.

Saeki, M. and Hori, M. (2006). Development of an Accurate Positioning System Using Low-Cost L1 GPS Receivers. Computer-Aided Civil and Infrastructure Engineering, 21(4), 258-267.

Schwieger, V. and Gläser, A. (2005). Possibilities of Low Cost GPS Technology for Precise Geodetic Applications. In: Proc. of the FIG Working Week 2005, Cairo, Egypt, Aprill 6-21, 2005. 
Söderholm, S. (2005). GPS L1 Carrier Phase Double Difference Solution Using Low Cost Receivers. In: Proc. of the ION GNSS 18th International Technical Meeting of the Satellite Division, Long Beach, CA., USA, 376-380.

Thales (2005). A12, B12 \& AC12 Reference Manual. USA.

van Bree, R.J.P. and Tiberius, C.C.J.M. (2012). Real-Time Single-Frequency Precise Point Positioning: Accuracy Assessment. GPS Solutions, 16 (2), 259-266.

Weston, N.D. and Schwieger, V. (2010). Cost Effective GNSS Positioning Techniques. FIG Commission 5 Publications, No. 49.

Zumberge, J.F., Heflin, M. B., Jefferson, D. C., Watkins, M. M. and Webb, F. H. (1997). Precise Point Positioning for the Efficient and Robust Analysis of GPS Data from Large Networks, Journal of Geophysical Research, 102 (B3), 5005-5017.

URL-1, The Precise Point Positioning Software Centre Web Page, Free Online PPP Services. http://gge.unb. ca/Resources/PPP/OnlinePPPs.html, July 11, 2012.

URL-2, NRCan, Online GPS/GNSS Post-Processing Service (CSRS-PPP) Web Page. http://www.geod. nrcan.gc.ca/online_data_e.php, July 11, 2012. 